\title{
Title: Central Role for Marginal Zone B Cells in an Animal Model of Sjogren's Syndrome
}

Long Shen*, Chun Gao*^, Lakshmanan Suresh†, Zhenhua Xian^, Nannan Song^, Lee D. Chaves*, Meixing Yu*, and Julian L. Ambrus Jr**

*Department of Medicine, Division of Allergy, Immunology and Rheumatology, SUNY at Buffalo School of Medicine and Biomedical Sciences

${ }^{\wedge}$ Department of Gastrointestinal Surgery, Tongji Hospital, Tongji Medical College, Huazhong University of Science and Technology, China

tDepartment of Oral Diagnostics Sciences, SUNY at Buffalo School of Dental

Medicine

Address Correspondence to:

Julian L. Ambrus Jr., MD

Division of Allergy, Immunology and Rheumatology

Room C281, Buffalo General Hospital

100 High Street

Buffalo, NY 14203

Phone: 716-859-2995

Fax: 716-859-1249

E-mail: jambrus@buffalo.edu

${ }^{\wedge}$ Long Shen, $\mathrm{PhD}$ and Chun Gao contributed equally to this paper.

Running Title: Marginal Zone B Cells in Sjogren's Syndrome

C 2016. This manuscript version is made available under the Elsevier user license 
This work was supported in part NATIONAL INSTITUTE OF DENTAL \&

CRANIOFACIAL RESEARCH grant 1R21DE021801-01A1 (to J.A.) and a grant NSFC81272623 from Surface Project of National Science Foundation of China (to C.G.) 


\begin{abstract}
Patients with Sjogren's syndrome (SS) have been shown to have abnormal B cell function and increased numbers of marginal zone B cells (MZB and MZB precursors. The current studies utilized the Interleukin 14 alpha transgenic mouse model (IL14aTG) for SS to investigate the roles of marginal zone B cells (MZB) of the innate immune system in the pathophysiology of the disease. Eliminating MZB from IL14aTG mice by B cell specific deletion of RBP-J resulted in complete elimination of all disease manifestations of SS. Mice had normal salivary gland secretions, negative autoantibodies and normal histology of the salivary and lacrimal glands compared to IL14aTG mice at the same time points. In contrast, eliminating B1 cells by deleting $b t k$ did not ameliorate the disease. Therefore, MZB are critical for the development of SS.
\end{abstract}




\section{INTRODUCTION}

Sjögren's syndrome (SS) is a common autoimmune disease in which loss of lachrymal gland function results in dry eyes, corneal abrasions and various other forms of eye inflammation and salivary gland injury leading to dry mouth, induction of dental caries and other oral infections. Systemic autoimmune manifestations include lymphocytic interstitial pneumonia, kidney disease especially renal tubular acidosis and eventually large B cell lymphomas in $5 \%$ of patients $(1,2)$. Sjögren's syndrome with lachrymal gland and salivary gland injury may be seen in conjunction with various other autoimmune diseases, including systemic lupus erythematosus (SLE) and rheumatoid arthritis. Current therapy is inadequate because lachrymal gland and salivary gland injury is advanced by the time symptoms are present and little is known regarding the pathophysiology of the disorder, especially the early events that may be amenable to therapeutic intervention.

Recent studies have suggested that B cells are central to the pathophysiology of SS (3-5). Increased numbers of marginal zone B cells (MZB) and MZB precursors have been noted in patients with SS $(6,7)$. Elimination of MZB from BAFF transgenic mice, a model of SS, eliminated salivary and lacrimal gland pathology in the mice (8). In the current studies we investigated particular roles for marginal zone B cells (MZB) in SS. We utilized the Interleukin 14 alpha transgenic (IL14aTG) mouse model of SS to evaluate the roles of MZB in SS. Work with this model, which has 
largely been corroborated in studies with human patients, has revealed that: 1) decrease in salivary gland flow precedes the infiltration of the salivary glands with lymphocytes (9); 2) deposition of autoantibodies on the salivary glands alone is not sufficient to cause salivary gland injury $(10) ; 3)$ autoantibodies to novel autoantigens, salivary protein 1 (SP-1), carbonic anhydrase 6 (CA6) and parotid secretory protein (PSP) are found far earlier in the course of the disease than antibodies to Ro, the autoantibody currently used in the diagnostic criteria for S (11); 4)lymphotoxin alpha (LTO) is critical for salivary gland injury, lung injury, kidney injury and lymphoma in SD (10); and 5) type 1 interferon (IFN-?] serves to amplify immune dysregulation after LT? induced injury to the submandibular and lachrymal glands - IL140TG mice lacking the type I interferon receptor develop submandibular and lachrymal gland injury but not parotid gland injury, lung injury or kidney injury. The development of lymphoma is very delayed in these mice (12). To investigate the role of MZB in SS we developed IL14aTG mice lacking MZB by crossing IL14aTG mice with C57BL/6. CD19Cre. RBP-J-/- mice, obtained from Dr. Tasuku Honjo, Kyoto University. The C57BL/6.CD19Cre.RBP-J-/- mice lack MZB but are otherwise immunologically normal (13). At the same time we developed IL14aTG mice lacking B1 cells by crossing IL14aTG mice with C57BL/6.btk-/- mice obtained from Dr. Wasif Khan. The C57BL/6. Btk-/- mice lack B1 cells but have normal B2 cells and MZB (14). 


\section{MATERIALS AND METHODS}

\section{Genetically Altered Mice}

The IL14aTG mice have previously been described (15). IL14aTG mice lacking MZB were generated by crossing IL14aTG mice with CD19Cre.RBP-J -/- mice obtained from Tasuka Honjo (13). IL14aTG mice lacking B1 cells were generated by crossing IL14aTG mice with C57BL/6.btk-/- mice obtained from Dr. Wasif Khan. The C57BL/6. Btk-/- mice lack B1 cells but have normal B2 cells and MZB (14). All mice were housed in Laboratory Animal Facility at University at Buffalo in accordance with institutional guidelines.

\section{Evaluation of Salivary Gland Secretions}

Salivary gland secretions were determined by suctioning of saliva for 15 minutes after Pilocarpine injection, as previously described (16).

\section{ELISA Assays for Autoantibodies}

Evaluation of anti-SP1 and anti-PSP antibodies in the sera of the mice were determined by Commercially available ELISA assays using recombinant murine SP1 and PSP antigens (IMMCO Diagnostics, NY), according to the manufacturer's instructions. 


\section{ELISA Assay for Interferon alpha and Lymphotoxin alpha}

Commercially available ELISA's were utilized to determine the serum levels of Interferon alpha (Life Technologies, Grand Island, NY) and Lymphotoxin alpha (R\&D Systems, Minneapolis, MN) according to the manufacturer's instructions.

\section{Flow Cytometry}

Flow cytometry was performed on mouse spleen cells treated with Zombie Aqua Fixable viability kit (BioLegend) blocked with TruStain fcX anti-mouse CD16/32 and stained with panels composed of anti-mouse antibodies from BioLegend (Alexa Fluor 700 anti-mouse IgD, APC anti-mouse B220, APC/Cy7 antimouse IgM, Brilliant violet421 anti-mouse CD-3, Brilliant violet 650 anti-mouse CD19, Brilliant Violet 785 anti-mouse CD25, FITC anti-mouse CD44, PE anti-mouse CD62L, PE/Cy5 anti-mouse CD4, PE/Cy7 anti-mouse NK-1.1, PE/Dazzle 594 antimouse CD8, PerCP/Cy5.5 anti-mouse CD21/35,) and Becton-Dickinson (BV605 rat anti-mouse CD5, BV711 rat anti-mouse CD23, Analysis was performed using a BD Biosciences LSRII Fortessa machine and FlowJo software.

\section{Isolation of Marginal Zone B cells (MZB).}


Spleens were first harvested from either IL-14a TG mice or C57/BL6 mice at age of 6 months old. Marginal Zone B cells were then purified using mouse Marginal Zone and Follicular B cell isolation kit from Miltenyi Biotec (San Diego, CA) following manufacturer instruction. The purities of MZB cells were confirmed by Flow Cytometry at range of $85-90 \%$.

\section{Quantitative PCR for the evaluation of LTA}

Quantitative PCR (qPCR) reaction was set up using SYBR select Master Mix purchased from Life Technologies (Carlsbad, CA) following the manufacturer's instructions. qPCR was run on an Stepone plus realtime PCR system (Life Technologies, Carlsbad, CA) with the following program: $50^{\circ} \mathrm{C}$ for $2 \mathrm{~min}$ hold, $95^{\circ} \mathrm{C}$ for 10 min hold, 40 cycles of $95^{\circ} \mathrm{C}, 15 \mathrm{~s}$ and $60^{\circ} \mathrm{C}$, $60 \mathrm{~s}$. Melting curve analysis was performed as previously described (4). The primers for LTA were forward primer 5'-CACGAGGTCCAGCTCTTTTC3' and reverse primer 5'-AGTGCAAAGGCTCCAAAGAA-3'; and for 18S rRNA control were forward primer 5'-CGCGGTTCTATTTTGTTGGT-3' and reverse primer 5'AGTCGGCATCGTTTATGGTC-3'. 


\section{RESULTS}

For the purposes of these studies colonies of ten C57BL/6, IL14a TG mice, IL14aTG.CD19Cre.RBP-J-/- and IL14aTG.btk-/- were followed for a period of 18 months. We utilized flow cytometry to evaluate the presence of various lymphocyte subpopulations in IL14aTG.CD19Cre.RBP-J-/- mice and IL14aTG mice. Figure 1a (IL14aTG mouse) and 1B (IL14aTG.CD19Cre.RBP-J-/- mouse) demonstrate representative flow panels that were performed on 6 mice in each group. Figure 1c summarizes the results. As previously published, the IL14aTG.CD19Cre.RBP-j-/mice lack MZB (The difference compared to IL14aTG, 27.1\% v 0.9\%, is statistically significant, $\mathrm{p}<0.001)(13)$. At the same time, compared to the IL-14aTG mice, the IL14aTG.CD19Cre.RBP-j-/- mice showed a slight decrease in the total number of B cells $(41.3 \% \mathrm{v} 23.5 \% ; \mathrm{p}=.006)$ and NK cells (29.2\% v 14.3\%; $\mathrm{p}=.0078)$, but similar numbers of CD4 cells, CD8 cells, Treg cells and double negative T cells. Studies published by Tanigaki et. Al. demonstrated that C57BL/6.CD19Cre.RBP-J-/- mice had normal T cells and NK cells function, as well as normal B cell function, except for the lack of MZB $(13,17)$.

Previous studies demonstrated that IL14aTG mice develop reduced salivary gland secretions by 6 months of age, before lymphocytic infiltration of the parotid glands is noted (9). We therefore investigated salivary gland secretions in IL14aTG mice and IL14aTG.CD19Cre.RBP-J-/- and compared them with IL14aTG.btk-/- mice 
at 6 and 12 months of age. Each group contained 6 mice. Figure 2 demonstrates that IL14aTG mice have reduced salivary gland secretions compared to C57BL/6 mice by 6 months of age (8.7 $\mu \mathrm{L} / \mathrm{Kg} \vee 11 \mu \mathrm{L} / \mathrm{Kg} ; \mathrm{p}=.0032)$, as previously published $(9,16)$. The IL14aTG.btk-/- mice also had significantly reduced salivary gland secretions compared to C57BL/6 mice $(7.2 \mu \mathrm{L} / \mathrm{Kg}$ v $11 \mu \mathrm{L} / \mathrm{Kg} ; \mathrm{p}<.001)$. The difference in salivary gland secretions between IL14aTG mice and IL14aTG.btk-/- mice was not statistically significant at this time point $(\mathrm{p}=.091)$. Interestingly, the salivary gland secretions of the IL14aTG.CD19Cre.RBP-J-/- were no different than the C57BL/6 mice at 6 months of age $(10.7 \mu \mathrm{L} / \mathrm{Kg} \mathrm{v} 11 \mu \mathrm{L} / \mathrm{Kg} ; \mathrm{p}=.417)$. At the 12 months time point, the difference between the salivary gland secretions of C57BL/6 mice and IL14ⓉG mice (9.3 $\mu \mathrm{L} / \mathrm{Kg}$ v $5.8 \mu \mathrm{L} / \mathrm{Kg}$; $\mathrm{p}=.0002)$ or IL14⿴TG.btk-/- mice (4.9 $\mu \mathrm{L} / \mathrm{kg} ; \mathrm{p}<.0001$ ) became even more significant. There was still no statistically significant difference between the salivary gland secretions of C57BL/6 mice and IL14aTG.CD19Cre.RBP-J-/- (9.3 $\mu \mathrm{L} / \mathrm{Kg}$ v $9.4 \mu \mathrm{L} / \mathrm{Kg} ; \mathrm{p}=.767)$.

We next investigated whether the changes in salivary gland secretions were followed by lymphocytic infiltration of the salivary and lachrymal glands. Previous studies have demonstrated that lymphocytic infiltration of the submandibular and lachrymal glands occurs before lymphocytic infiltration of the parotid glands (18). As demonstrated in Figure 3, IL14aTG.CD19Cre.RBP-J-/- never developed lymphocytic infiltration of either the submandibular or parotid glands. The IL14aTG.btk-/- mice, like the IL14aTG mice, had lymphocytic infiltration of both the submandibular and parotid glands by 12 months of age. No lymphocytic infiltration 
is seen in the salivary glands in control C57BL/6 mice. This is representative of 12 mice studied in each group;

Decreased salivary gland function is also associated with production of autoantibodies to salivary gland antigens. Previous studies demonstrated that antibodies to salivary gland protein 1 (SP1) and parotid secretory protein (PSP) occur before antibodies to Ro and La (11).We therefore investigated the presence of these autoantibodies in the sera of C57BL/6, IL14a TG mice and IL14aTG.CD19Cre.RBP-J-/- mice at 6 and 12 months of age. Six mice were studied in each group. Figure 4 demonstrates that these autoantibodies were present in IL14aTG mice but not in either C57BL/6 mice or IL14aTG.CD19Cre.RBP-J-/- mice at either time point. The difference in serum SP1 autoantibodies between IL14aTG mice and IL14aTG.CD19Cre.RBP-J-/- mice was statistically significant at both 6 months $(.20 \mathrm{v} .02 ; \mathrm{p}=.0019)$ and 12 months of age $(.26 \mathrm{v} .01 ; \mathrm{p}=.00002)$ as was the difference in serum PSP antibodies at 6 months $(.21 \mathrm{v} .004 ; \mathrm{p}=.017)$ and 12 months $(.28 \mathrm{v} .05 ; \mathrm{p}=.0056)$ of age. The serum antibodies to SP1 were not statistically different between C57BL/6 mice and IL14aTG.CD19Cre.RBP-J-/- mice at either 6 months $(\mathrm{p}=.055)$ or 12 months $(\mathrm{p}=.111)$ of age similar to the serum levels of PSP antibodies at 6 months $(\mathrm{p}=.667)$ and 12 months $(\mathrm{p}=.377)$ of age.

Early injury to the submandibular and lachrymal glands in SS has been associated with increased levels of LTa in the serum and salivary gland secretions 
(10). We therefore investigated the levels if LTa in the sera of C57BL/6, IL14a TG mice, IL14aTG.CD19Cre.RBP-J-/- and IL14aTG.btk-/- mice. Figure 5 demonstrates that serum levels LTa were elevated in sera of IL14aTG mice $(2554 \mathrm{ng} / \mathrm{ml})$ and IL14aTG.btk-/- mice (3495ng/ml) compared to C57BL/6 mice (1869 ng/ml) at both 7 months (IL14aTG, $\mathrm{p}=.0363$; IL14aTG.btk-/- $\mathrm{p}=.0032$ ) and 12 months of age $($ IL14aTG $=3859 \mathrm{ng} / \mathrm{ml}, \mathrm{p}=.0104 ;$ IL14aTG.btk- $/-5652 \mathrm{ng} / \mathrm{ml} ; \mathrm{p}=.0024)$ while the serum levels of LTa in IL14aTG.CD19Cre.RBP-J-/- were no different than the C57BL/6 controls at either 7 months (1926 ng/ml v $1869 \mathrm{ng} / \mathrm{ml}$; $\mathrm{p}=.824)$ or 12 months of age ( $2086 \mathrm{ng} / \mathrm{ml} v 2181 \mathrm{ng} / \mathrm{ml} ; \mathrm{p}=.7022)$. Six mice were studied in each group;

Because MZB have been shown to produce various cytokines including LTa (19), we investigated whether MZB isolated from IL14aTG mice constitutively produced LTa at the 6 month time point when salivary gland injury is occurring in these mice. Figure 6 demonstrates that MZB from IL14aTG expressed the mRNA for LTa at significantly higher levels $(2.88 \mathrm{v} 1.01 ; \mathrm{p}<.0001)$ than C57BL/ 6 mice at the same age. In data not shown, production of IL-6, IL-10 and TNF-a was not detected in either the MZB of IL14aTG mice or C57BL/6 mice. Six mice were studies in each group.

Later injury to the parotid glands, lungs and kidney in SS has been associated with the production of IFN-a (12). We therefore investigated the serum levels of IFN-a in sera of C57BL/6, IL14a TG mice, IL14aTG.CD19Cre.RBP-J-/- and IL14aTG.btk-/- mice during the evolution of SS. Figure 7 demonstrates that IFN-a was undetectable in the sera of C57BL/6 mice $(0 \mathrm{pg} / \mathrm{ml})$ and IL14aTG.CD19Cre.RBP- 
J-/- mice $(0 \mathrm{pg} / \mathrm{ml})$ at 12 months of age, while they were elevated in both IL14aTG mice (422 pg/ml) and IL14aTG.btk-/- mice (411 pg/ml) (compared to C57BL/6 mice, $\mathrm{p}<0.001$ for both IL14aTG and IL14aTG.btk-/- mice). The difference in the serum levels of IFN-a in IL14aTG and IL14aTG.btk-/- mice was not statistically significant $(\mathrm{p}=.8848)$. Six mice were studied in each group.

\section{DISCUSSION}

While autoimmune diseases were once thought to be caused exclusively by abnormal recognition of self-antigens by the adaptive immune system, recent 
studies have emphasized the importance of the innate immune system especially early in the course of the disease (20-22). Patients with SS are generally recognized at late stages of the disease, so animal models, such as the IL14aTG mouse, have been critical in elucidating early stages in disease pathogenesis (23). Previous studies with this animal model have emphasized the importance of LTa during the initial stages of the disease and IFN-a in a later stage of the disease, as animals go from manifestations localized to the lacrimal and salivary glands to systemic manifestations including lung disease and lymphoma $(10,12)$. The current studies emphasize the importance of MZB in disease pathogenesis demonstrating the absence of disease in IL14aTG mice lacking MZB. MZB are considered part of the innate immune system because of their limited repertoire and their functioning largely independently of T cell help $(19,24)$. However, MZB may also function as part of the adaptive immune system carrying antigens to the germinal centers for more efficient generation of memory B cells in the B2 compartment (25). At the same time, MZB may be responsible for the majority of IgM memory B cells $(26,27)$. Differences exist between MZB in rodents and humans, one of which is increased circulation of human MZB compared to rodent MZB $(26,27)$. These differences may make MZB more important rather than less important for the pathophysiology of SS in humans (5). In the current studies we demonstrate that IL14aTG mice lacking MZB (Figure 1) had normal salivary gland secretions (Figure 2), normal salivary and lacrimal gland histology (Figure 3), absence of autoantibody production (Figure 4) and reduced levels of both LTa and IFN-a (Figures 5 and 7). MZB from IL14aTG 
mice were shown to produce LTa (Figure 6). No lung, disease or lymphoma was seen in these mice.

Various self-reactive antibodies are produced by MZB. When a transgene was expressed making anti-DNA antibody in mice, the anti-DNA producing B cells were found in the marginal zones and had the characteristics of MZB (28). They have been found in the salivary glands of patients with SS (29). Because MZB require weak signaling through their B cell receptors, they are more adept at responding to pathogens than to self-antigens (19). MZB express MHC II and B7.1 constitutively, allowing them to present antigen efficiently to T cells (30). MZB can secrete IL-10 and act as regulatory B cells, thus inhibiting rather than stimulating various $\mathrm{T}$ cell responses (31). Alternatively, MZB can serve to focus the T cell response to particular pathogens to restricted locations. MZB may contribute to autoimmunity in the salivary glands by reacting to pathogens in the salivary glands and keeping the response local. In the IL14aTG mouse, SS does not develop in the absence of MZB. The development of SS is a complex process likely requiring multiple immunological changes and the contributions of MZB in this process are essential. Studies in patients with SS have also identified MZB as prominent in the disease (32, 33). These and other factors have made B cells therapeutic targets in SS $(34,35)$.

Overall, these studies have provided novel insights into the role of innate B cells in the pathophysiology of SS in IL14aTG mice. In a similar animal model for SS, the BAFF transgenic mouse, MZB have been found to be central to the development 
of SS $(8,36)$. Interestingly, however, in this animal model, which develops a more proliferative glomerulonephritis than the IL14aTG mouse, B1 cells were shown to be critical for the development of the glomerulonephritis. Elimination of MZB from BAFF transgenic mice does not influence the glomerulonephritis. This raises two interesting points, first that the involvement of the innate immune system and particular innate B cells may be different in various autoimmune diseases and second that in an animal with an autoimmune disease involving multiple different manifestations, the nature of injury in various organs may be different. It would be interesting in the IL14aTG mouse to eliminate MZB at an intermediate or late stage of disease and see whether late stage manifestations are inhibited after early stage injury is allowed to occur. The data from the IL14aTG mouse continue to support the hypothesis that SS evolves through stages of local disease driven by LTa, followed by systemic disease driven by IFN-a followed by malignancy. However, it is also possible that particular patients with SS could present with both local and systemic manifestations while others could have local manifestations and never develop systemic manifestations. Understanding these issues will have major importance in determining optimal therapies for patients with different types / stages of disease.

Acknowledgments

We would like to thank Drs Tasuka Honjo and Wasif Khan for providing the mice used in this study, and Christ Becker for technical assistance for pathology studies. 


\section{REFERENCES}

1. GarciaCarrasco, M., M. RamosCasals, J. Rosas, L. Pallares, J. CalvoAlen, R. Cervera, J. Font, and M. Ingelmo. 2002. Primary Sjogren syndrome - Clinical and immunologic disease patterns in a cohort of 400 patients. Medicine 81: 270-280.

2. Fox, R. I. 2005. Sjogren's syndrome. Lancet 366: 321-331.

3. Jonsson, R., E. Nginamau, E. Szyszko, and K. A. Brokstad. 2007. Role of B cells in Sjogren's syndrome - from benign lymphoproliferation to overt malignancy. Frontiers in Bioscience 12: 2159-2170.

4. Kroese, F. G. M., W. H. Abdulahad, E. Haacke, N. A. Bos, A. Vissink, and H. Bootsma. 2014. B-cell hyperactivity in primary Sjogren's syndrome. Expert Review of Clinical Immunology 10: 483-499.

5. Pers, J. O., and P. Youinou. 2014. Are the B cells cast with the leading part in the Sjogren's syndrome scenario? Oral Diseases 20: 529-537.

6. Daridon, C., J. O. Pers, V. Devauchelle, C. MartinsCarvalho, P. Hutin, Y. L. Pennec, A. Saraux, and P. Youinou. 2006. Identification of transitional type IIB cells in the salivary glands of patients with Sjogren's syndrome. Arthritis and rheumatism 54: 2280-2288.

7. Vossenkamper, A., P. M. K. Lutalo, and J. Spencer. 2012. Translational MiniReview Series on B cell subsets in disease. Transitional B cells in systemic lupus erythematosus and Sjogren's syndrome: clinical implications and effects of B cell-targeted therapies. Clin Exp Immunol 167: 7-14.

8. Fletcher, C. A., A. P. Sutherland, J. R. Groom, M. L. Batten, L. G. Ng, J. Gommerman, and F. Mackay. 2006. Development of nephritis but not sialadenitis in autoimmune-prone BAFF transgenic mice lacking marginal zone B cells. Eur J Immunol 36: 2504-2514.

9. Shen, L., L. Suresh, H. Li, C. Zhang, V. Kumar, O. Pankewycz, and J. J. Ambrus. 2009. IL-14alpha, the Nexus for Primary Sjogren's Disease in Mice and Humans. Clinical immunology 130: 304-312.

10. Shen, L., L. Suresh, J. Wu, J. X. Xuan, H. Li, C. J. Zhang, O. Pankewycz, and J. L. Ambrus. 2010. A Role for Lymphotoxin in Primary Sjogren's Disease. Journal of immunology 185: 6355-6363.

11. Shen, L., L. Suresh, M. Lindemann, J. Xuan, P. Kowal, K. Malyavantham, and J. L. Ambrus, Jr. 2012. Novel autoantibodies in Sjogren's syndrome. Clinical immunology 145: 251-255.

12. Shen, L., L. Suresh, K. Malyavantham, P. Kowal, J. X. Xuan, M. J. Lindemann, and J. L. Ambrus. 2013. Different Stages of Primary Sjogren's Syndrome Involving Lymphotoxin and Type 1 IFN. Journal of immunology 191: 608-613. 
13. Tanigaki, K., H. Han, N. Yamamoto, K. Tashiro, M. Ikegawa, K. Kuroda, A. Suzuki, T. Nakano, and T. Honjo. 2002. Notch-RBP-J signaling is involved in cell fate determination of marginal zone B cells. Nat Immunol 3: 443-450.

14. Khan, W. N., F. W. Alt, R. M. Gerstein, B. A. Malynn, I. Larsson, G. Rathbun, L. Davidson, S. Muller, A. B. Kantor, L. A. Herzenberg, and et al. 1995. Defective B cell development and function in Btk-deficient mice. Immunity 3: 283-299.

15. Shen, L., C. Zhang, T. Wang, S. Brooks, R. J. Ford, Y. C. Lin-Lee, A. Kasianowicz, V. Kumar, L. Martin, P. Liang, J. Cowell, and J. Ambrus, JL. 2006. Development of Autoimmunity in Interleukin-14alpha Transgenic Mice. Journal of immunology 177: 5676-5686.

16. Shen, L., L. Suresh, J. Wu, X. Jingxiu, H. Li, C. Zhang, O. Pankewycz, and J. J. Ambrus. 2010. A Role for Lymphotoxin in Primary Sjogren's Disease. J. Immunol. 185: 6355-6363.

17. Tanigaki, K., and T. Honjo. 2007. Regulation of lymphocyte development by Notch signaling. Nat Immunol 8: 451-456.

18. Xuan, J. X., L. Shen, K. Malyavantham, O. Pankewycz, J. L. Ambrus, and L. Suresh. 2013. Temporal histological changes in lacrimal and major salivary glands in mouse models of Sjogren's syndrome. Bmc Oral Health 13.

19. Pillai, S., A. Cariappa, and S. T. Moran. 2005. Marginal zone B cells. Annual review of immunology 23: 161-196.

20. Allam, R., and H. J. Anders. 2008. The role of innate immunity in autoimmune tissue injury. Current Opinion in Rheumatology 20: 538-544.

21. Lehuen, A., J. Diana, P. Zaccone, and A. Cooke. 2010. Immune cell crosstalk in type 1 diabetes. Nature Reviews Immunology 10: 501-513.

22. Nikolov, N. P., and G. G. Illei. 2009. Pathogenesis of Sjogren's syndrome. Curr Opin Rheumatol 21: 465-470.

23. Donate, A., A. Voigt, and C. Q. Nguyen. 2014. The value of animal models to study immunopathology of primary human Sjogren's syndrome symptoms. Expert Review of Clinical Immunology 10: 469-481.

24. Woods, K. M., M. R. Pope, S. M. Hoffman, and S. D. Fleming. 2011. CR2(+) Marginal Zone B Cell Production of Pathogenic Natural Antibodies Is C3 Independent. Journal of immunology 186: 1755-1762.

25. You, Y., R. C. Myers, L. Freeberg, J. Foote, J. F. Kearney, L. B. Justement, and R. H. Carter. 2011. Marginal Zone B Cells Regulate Antigen Capture by Marginal Zone Macrophages. Journal of immunology 186: 2172-2181.

26. Weill, J. C., S. Weller, and C. A. Reynaud. 2009. Human Marginal Zone B Cells. Annual review of immunology 27: 267-285.

27. Weller, S., M. C. Braun, B. K. Tan, A. Rosenwald, C. Cordier, M. E. Conley, A. Plebani, D. S. Kumararatne, D. Bonnet, O. Tournilhac, G. Tchernia, B. Steiniger, L. M. Staudt, J. L. Casanova, C. A. Reynaud, and J. C. Weill. 2004. Human blood IgM "memory" B cells are circulating splenic marginal zone B cells harboring a prediversified immunoglobulin repertoire. Blood 104: 3647-3654.

28. Li, H., Y. Jiang, E. L. Prak, M. Radic, and M. Weigert. 2001. Editors and editing of anti-DNA receptors. Immunity 15: 947-957.

29. Le Pottier, L., V. Devauchelle, A. Fautrel, C. Daridon, A. Saraux, P. Youinou, and J. O. Pers. 2009. Ectopic Germinal Centers Are Rare in Sjogren's Syndrome 
Salivary Glands and Do Not Exclude Autoreactive B Cells. Journal of immunology 182: 3540-3547.

30. Attanavanich, K., and J. F. Kearney. 2004. Marginal zone, but not follicular B cells, are potent activators of naive CD4 T cells. Journal of immunology 172: 803-811.

31. Jamin, C., A. Morva, S. Lemoine, C. Daridon, A. R. de Mendoza, and P. Youinou. 2008. Regulatory B lymphocytes in humans - A potential role in autoimmunity. Arthritis and rheumatism 58: 1900-1906.

32. Mariette, X. 2008. Therapeutic Potential for B-Cell Modulation in Sjogren's Syndrome. Rheumatic Disease Clinics of North America 34: 1025-+.

33. Youinou, P., V. Devauchelle, P. Hutin, R. Le Berre, A. Saraux, and J. O. Pers. 2007. A conspicuous role for B cells in Sjogren's syndrome. Clinical reviews in allergy \& immunology 32: 231-237.

34. Alcantara, C., M. J. Gomes, and C. Ferreira. 2009. Rituximab Therapy in Primary Sjogren's Syndrome. In Contemporary Challenges in Autoimmunity. Y. Shoenfeld, and M. E. Gershwin, eds. 701-705.

35. Pijpe, J., G. W. vanImhoff, F. K. L. Spijkervet, J. L. N. Roodenburg, G. J. Wolbink, K. Mansour, A. Vissink, C. G. M. Kallenberg, and H. Bootsma. 2005. Rituximab treatment in patients with primary Sjogren's syndrome. Arthritis and rheumatism 52: 2740-2750.

36. Mackay, F., S. A. Woodcock, P. Lawton, C. Ambrose, M. Baetscher, P. Schneider, J. Tschopp, and J. L. Browning. 1999. Mice transgenic for BAFF develop lymphocytic disorders along with autoimmune manifestations. J Exp Med 190: 1697-1710.

\section{FIGURE LEGENDS}

\section{Figure 1}

Flow cytometry was performed on the spleens of 4 mice each of IL14aTG mice and IL14aTG.CD19Cre.RBP-j-/- mice at 6 months of age as outlined in materials and methods. Representative flow studies are shown for an IL14aTG 
mouse in Figure 1a, for an IL14aTG.CD19Cre.RBP-j-/- mouse in Figure 1b. The results are summarized in Figure 1c. The IL14aTG.CD19Cre.RBP-j-/- mice lack MZB (The difference compared to IL14aTG is statistically significant, $\mathrm{p}<0.001$ ). At the same time, compared to the IL-14aTG mice, the IL14aTG.CD19Cre.RBP-j-/- mice showed a slight decrease in the total number of B cells $(p=.006)$ and NK cells $(p=$ .0078), but similar numbers of CD4 cells, CD8 cells, Treg cells and double negative T cells. The bars on the top of the figure emphasize statistically significant differences.

\section{Figure 2}

This figure demonstrates the salivary gland secretions determined after Pilocarpine stimulations of groups of mice indicated as above. Ten mice were studied in each group at 6 and 12 months of age. The group designated IL $14 \alpha$ TG.btk- mice is IL14 $\alpha$ TG.btk-/- and the group designated IL14 $\alpha$ TG.RBP-J- is IL14 $\alpha$ TG.CD19Cre.RBP-J-

/-. The difference between the salivary gland secretions of C57BL/6 mice at 6 months of age and those of IL14 $\alpha$ TG mice at 6 months of age was statistically significant ( $p$ $=.0032)$ as was the difference between C57BL/6 mice at 6 months of age and IL14 $\alpha$ TG.btk-/- mice at 6 months of age $(p<.0001)$. There was no difference between the salivary gland secretions of C57BL/6 mice at 6 months of age and those of IL14 $\alpha$ TG.CD19Cre.RBP-J-/- mice at 6 months of age $(p=.4166)$ or of C57BL/6 mice and IL14 $\alpha$ TG.CD19Cre.RBP-J-/- mice at 12 months of age $(p=.767)$. At 12 months of 
age the difference between the salivary gland secretions of C57BL/6 mice and IL14 $\alpha$ TG mice $(p=.0002)$ or IL $14 \alpha$ TG.btk-/- mice $(p<.0001)$ became even more significant. The bars on the top of the figure emphasize statistically significant differences.

\section{Figure 3}

The figure demonstrates representative pictures of submandibular and

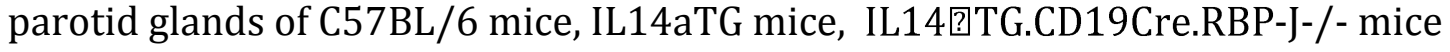
and IL14?TG.btk-/- mice at 12 months of age. Normal histology of the salivary glands was noted in 24/24 C57BL/6 mice and 24/24 IL14@TG.CD19Cre.RBP-J-/mice studied while abnormal histology was noted in 24/24 IL14aTG mice and 14/14 IL14⿴囗TG.btk-/- mice studied. The bars on the top of the figure emphasize statistically significant differences.

\section{Figure 4}

Sera were collected from IL-14国G mice, IL14aTG.CD19Cre.RBP-j -/- and C57BL/ 6 mice at 6 and 12 months of age. Custom expressed and purified salivary gland protein 1 (SP-1) and parotid secretory protein (PSP) were used to run ELISA assays with these sera. Data shown are representative of 6 mice studied in each group. The difference in serum SP1 autoantibodies between IL14aTG mice and IL14aTG.CD19Cre.RBP-J-/- mice was statistically significant at both 6 months ( $\mathrm{p}=$ 
$.0019)$ and 12 months of age $(p=.00002)$ as was the difference in serum PSP antibodies at 6 months $(p=.017)$ and 12 months $(p=.0056)$ of age. The levels of serum antibodies to SP1 were not statistically different between C57BL/6 mice and IL14aTG.CD19Cre.RBP-J-/- mice at either 6 months $(\mathrm{p}=.055)$ or 12 months ( $\mathrm{p}=$ .111) of age similar to the serum levels of PSP antibodies at 6 months ( $p=.667)$ and 12 months ( $\mathrm{p}=.377)$ of age. The bars on the top of the figure emphasize statistically significant differences.

\section{Figure 5}

LTa levels were determined by ELISA in the sera of C57BL/6 mice, IL14aTG mice, IL14aTG.CD19Cre.RBP-j -/- mice (labeled as IL14aTG.RBP-J-/-) and IL14aTG.BTK-/- mice at 7 and 12 months of age. Data shown are the serum levels of LTa in $\mathrm{ng} / \mathrm{ml}$ for each of the mice studied. The data used in this figure for the C57BL/6 mice and the IL14aTG mice were part of a previous publication, but are included here for the sake of completeness. The studies in the IL14aTG.CD19Cre.RBP-J-/- mice and IL14aTG.BTK-/- mice were done at the same time. Serum levels of LTa were significantly increased in IL14aTG (7M p=. 0363; $12 \mathrm{M} \mathrm{p}=.0104)$ and IL14aTG.BTK- $/-$ mice $(7 \mathrm{M} \mathrm{p}=.0032 ; 12 \mathrm{M} \mathrm{p}=.0024)$ compared to C57BL/6 mice at both time points studied, while the serum levels of LTa were statistically similar in IL14aTG.CD19Cre.RBP-J-/- mice $(7 \mathrm{M} \mathrm{p}=.824 ; 12 \mathrm{M} \mathrm{p}=.7022)$ compared to C57BL/ 6 mice at both time points studied. There was no statistically significant difference between the serum levels of LTa in IL14aTG mice compared to 
IL14aTG.BTK-/- mice at either time point studied (7M p = .0553; 12M p =.1127).

The bars on the top of the figure emphasize statistically significant differences.

\section{Figure 6}

Splenic marginal zone B cells were isolated from 6 month-old C57BL/6 and IL14aTG mice as outlined in Materials and Methods. qPCR was utilized to quantitate the mRNA for LTa in these B cells as outline in Materials and Methods. Data are shown are the relative levels of LTa mRNA in each of the mice studied. The difference in the level of LTa mRNA in C57BL/6 mice compared to IL14aTG mice was statistically significant $(\mathrm{P}<.0001)$. The bars on the top of the figure emphasize statistically significant differences.

\section{Figure 7}

Interferon alpha levels were determined by ELISA in the sera of C57BL/6 mice, IL14aTG mice, IL14aTG.CD19Cre.RBP-j -/- mice (labeled as IL14aTG.RBP-J-/-) and IL14aTG.BTK-/- mice at 12 months of age. Data shown are the serum levels of interferon alpha in $\mathrm{pg} / \mathrm{ml}$ for each of the mice studied. The differences between the interferon alpha levels of the sera from IL14aTG mice $(\mathrm{p}<0.001)$ and IL14aTG.BTK /- $(\mathrm{p}<0.001)$ compared to the C57BL/6 mice was statistically significant. The difference in the interferon alpha levels in the sera of IL14aTG mice compared to IL14aTG.BTK-/- mice was not statistically significant $(\mathrm{p}=.8848)$. The bars on the top of the figure emphasize statistically significant differences. 
Figure 1A

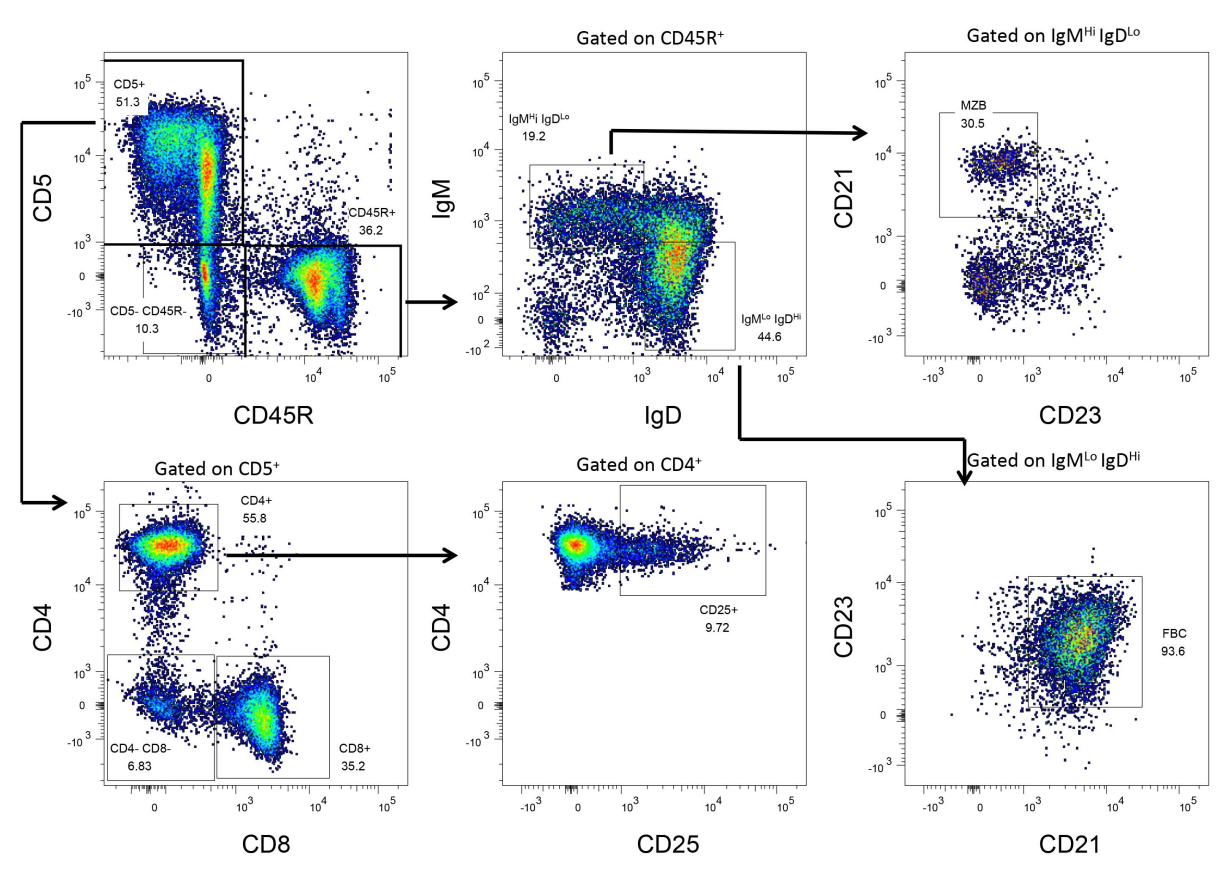

Figure 1B

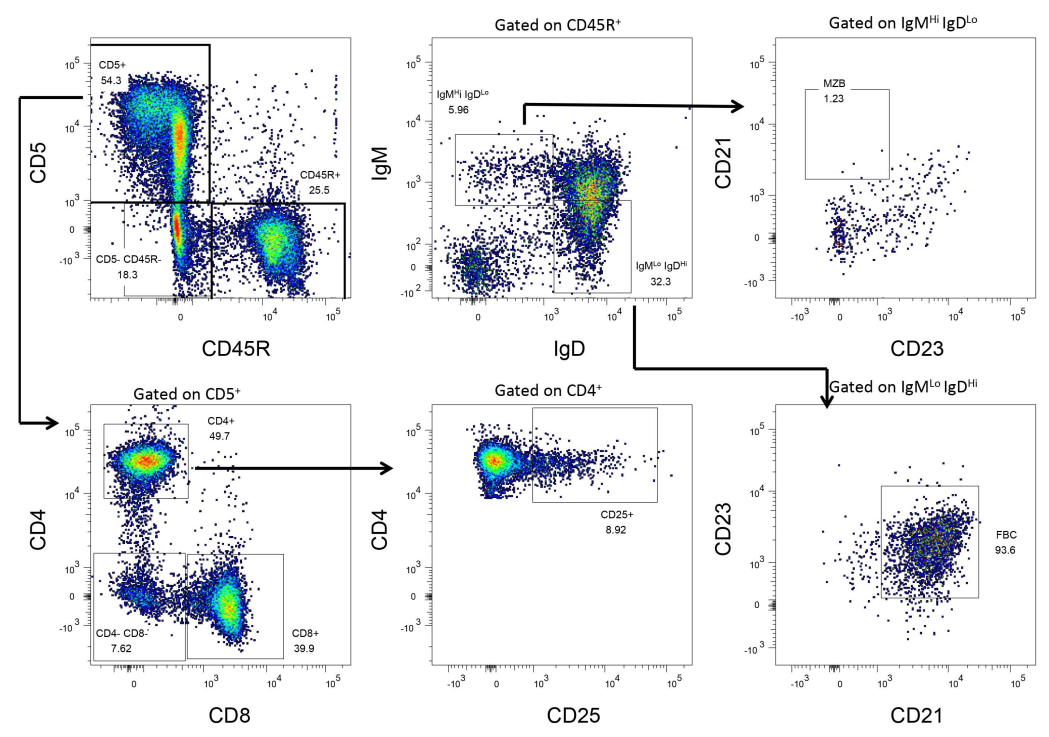


Figure 1C

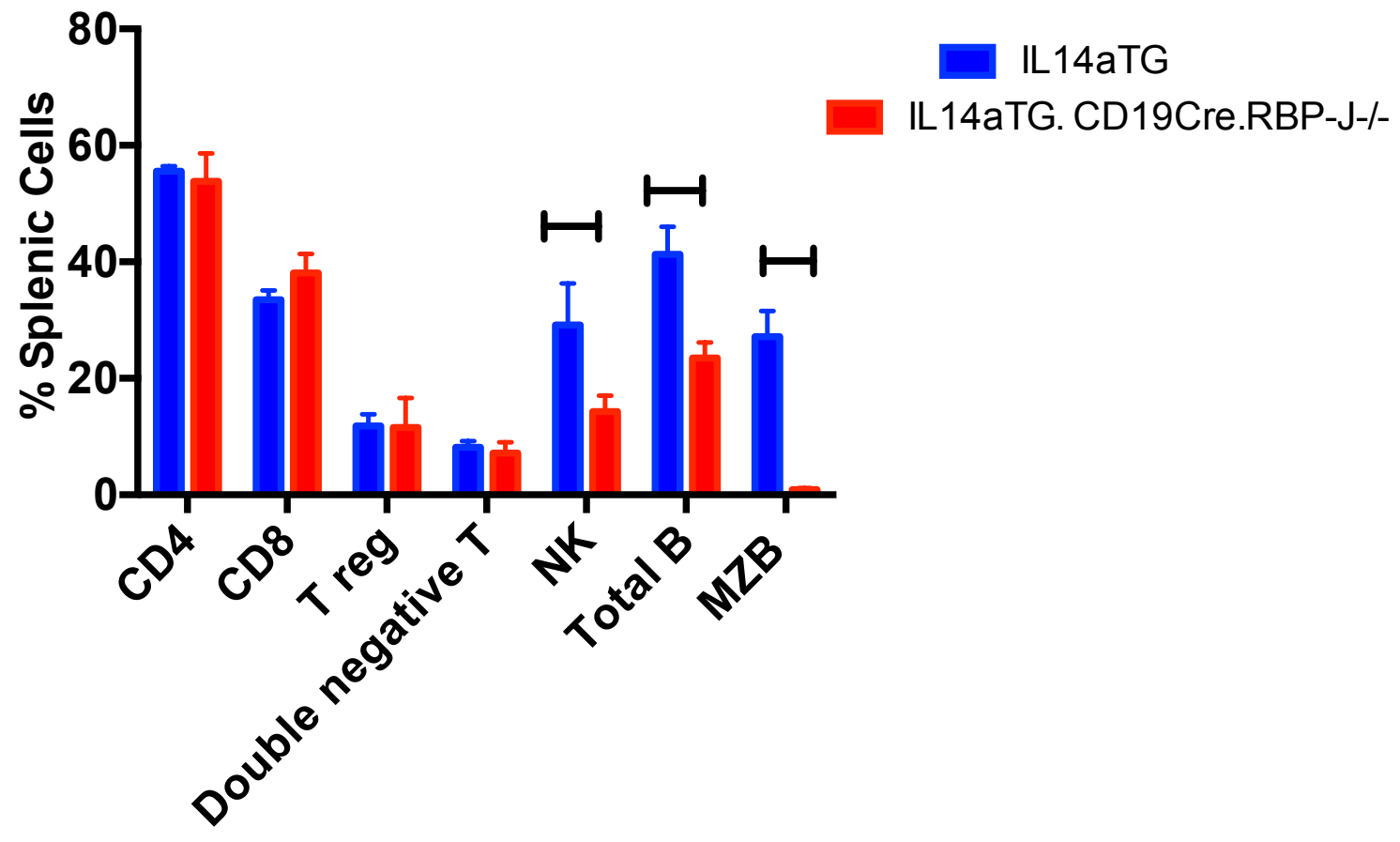


Figure 2

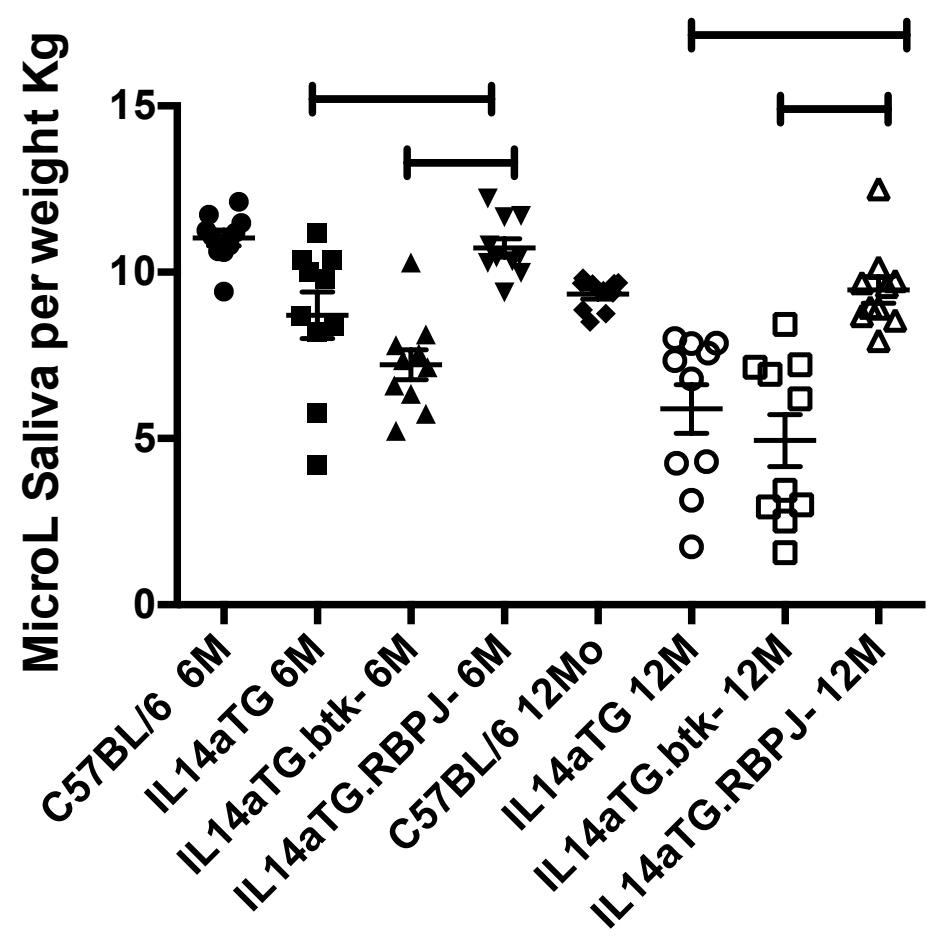


Figure 3

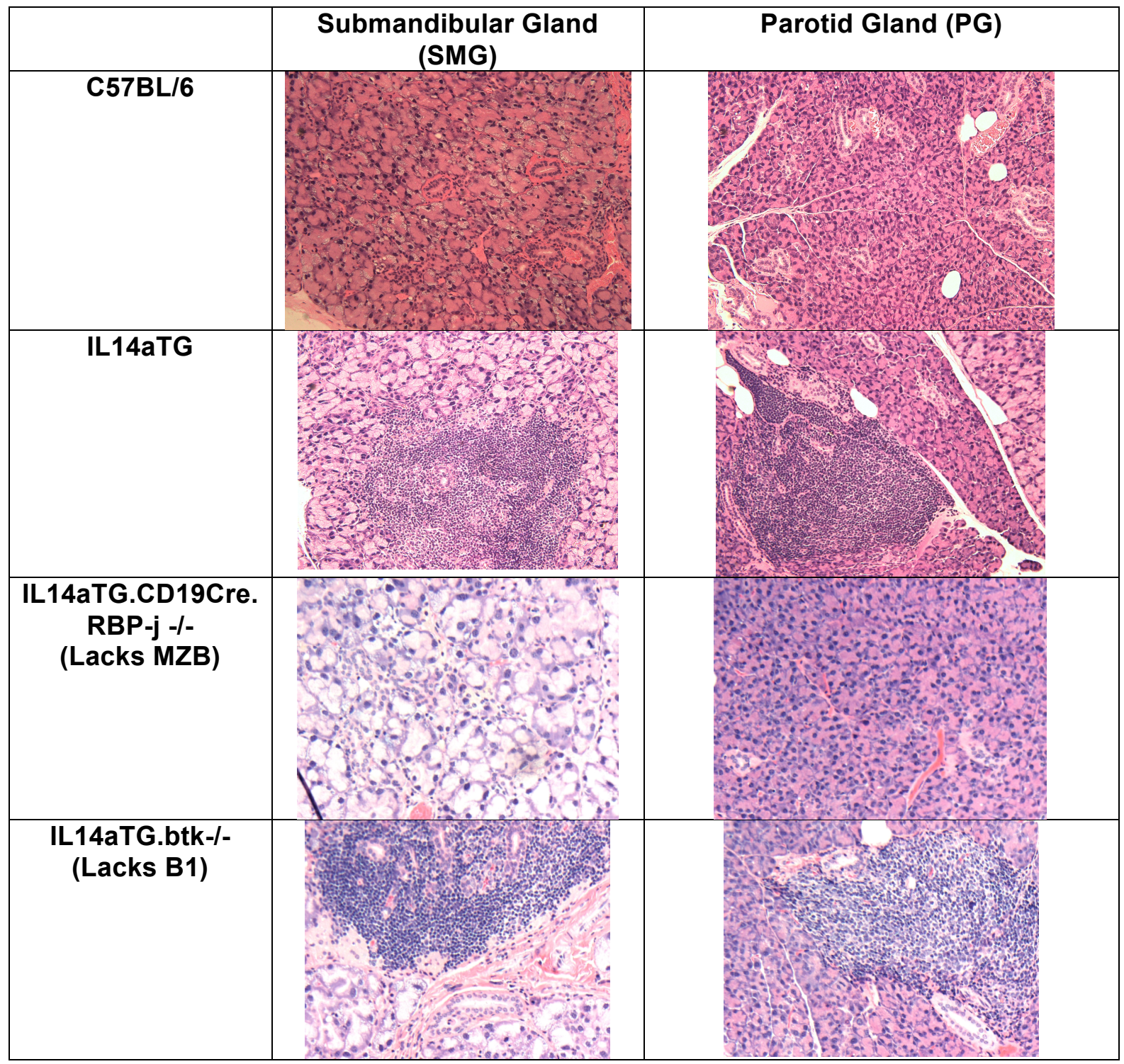


Figure 4

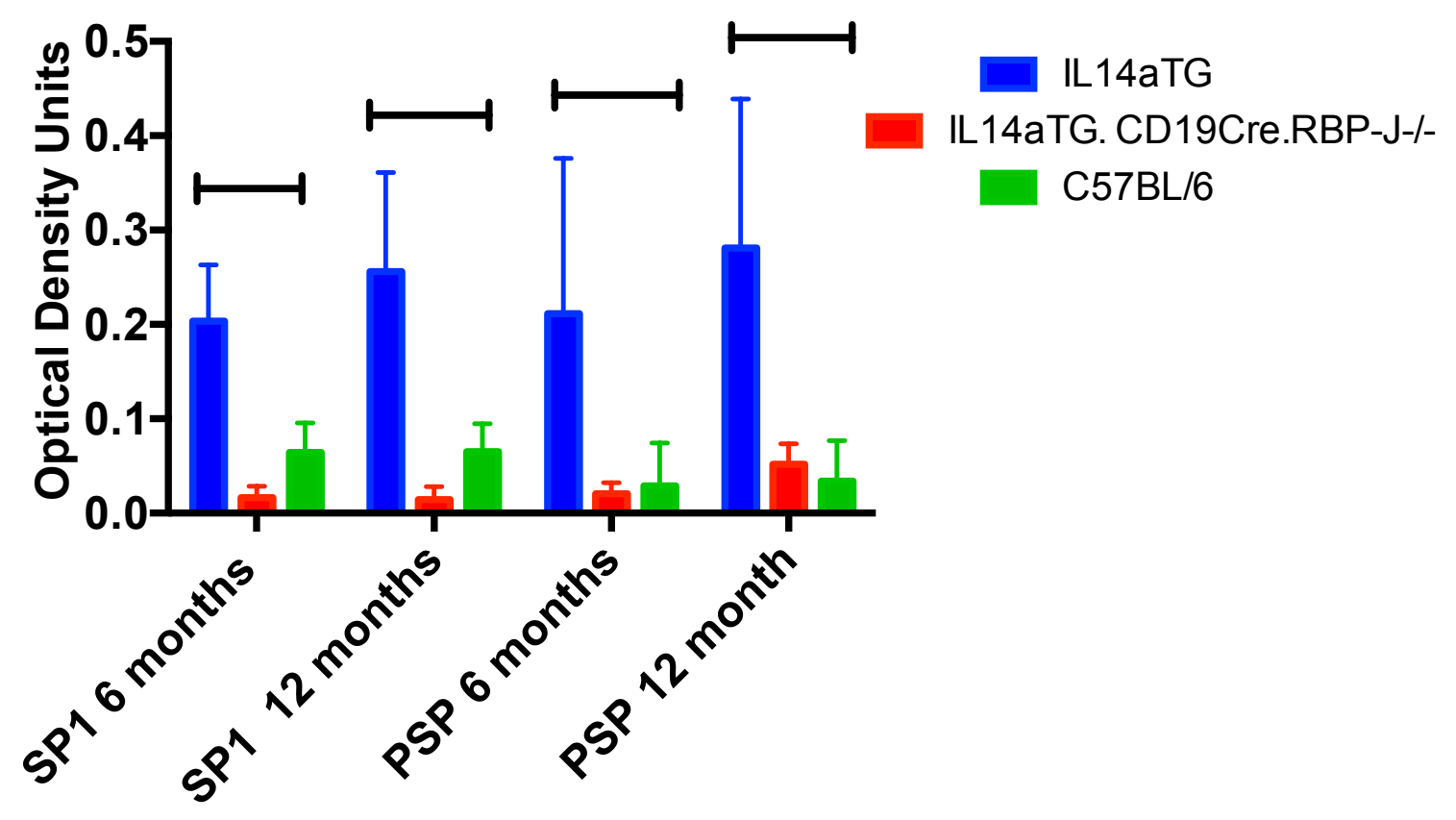


Figure 5

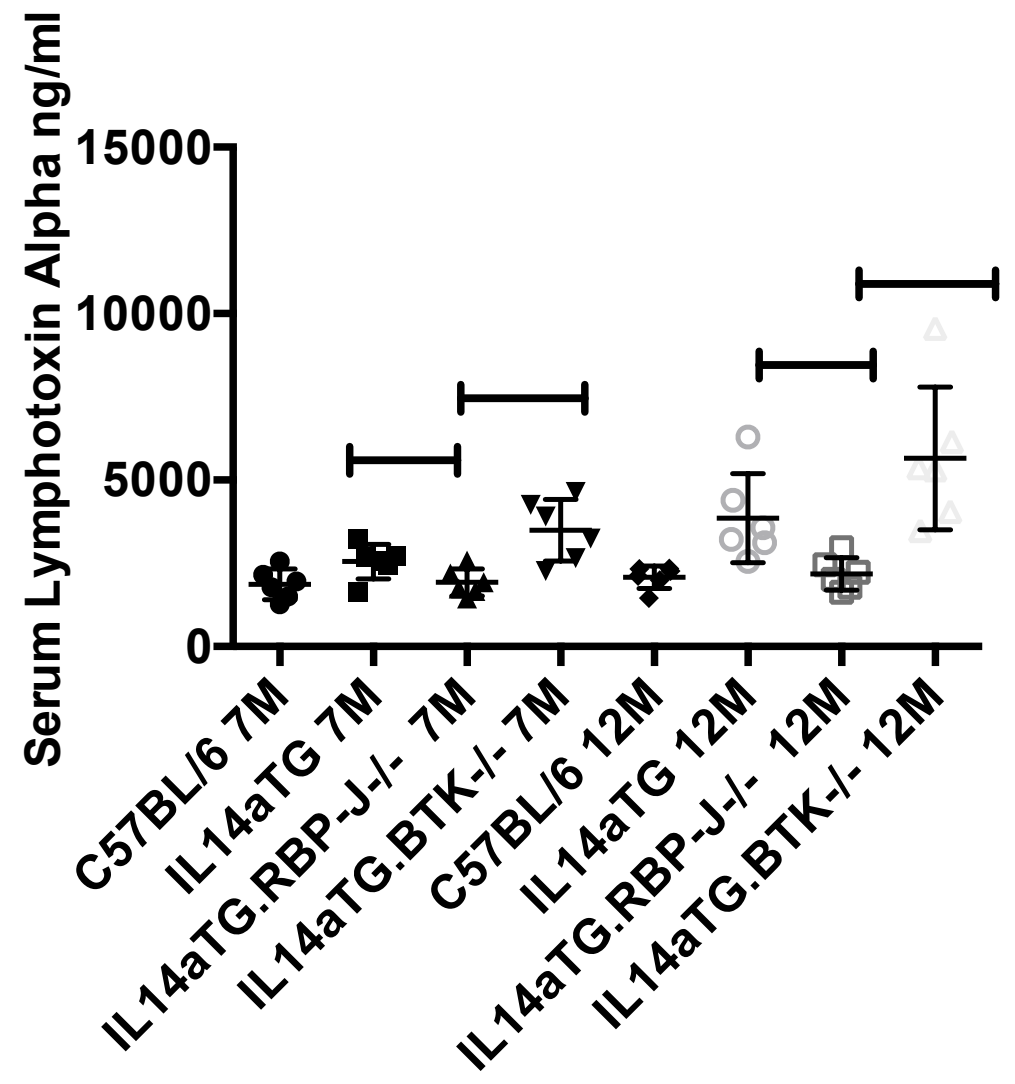


Figure 6

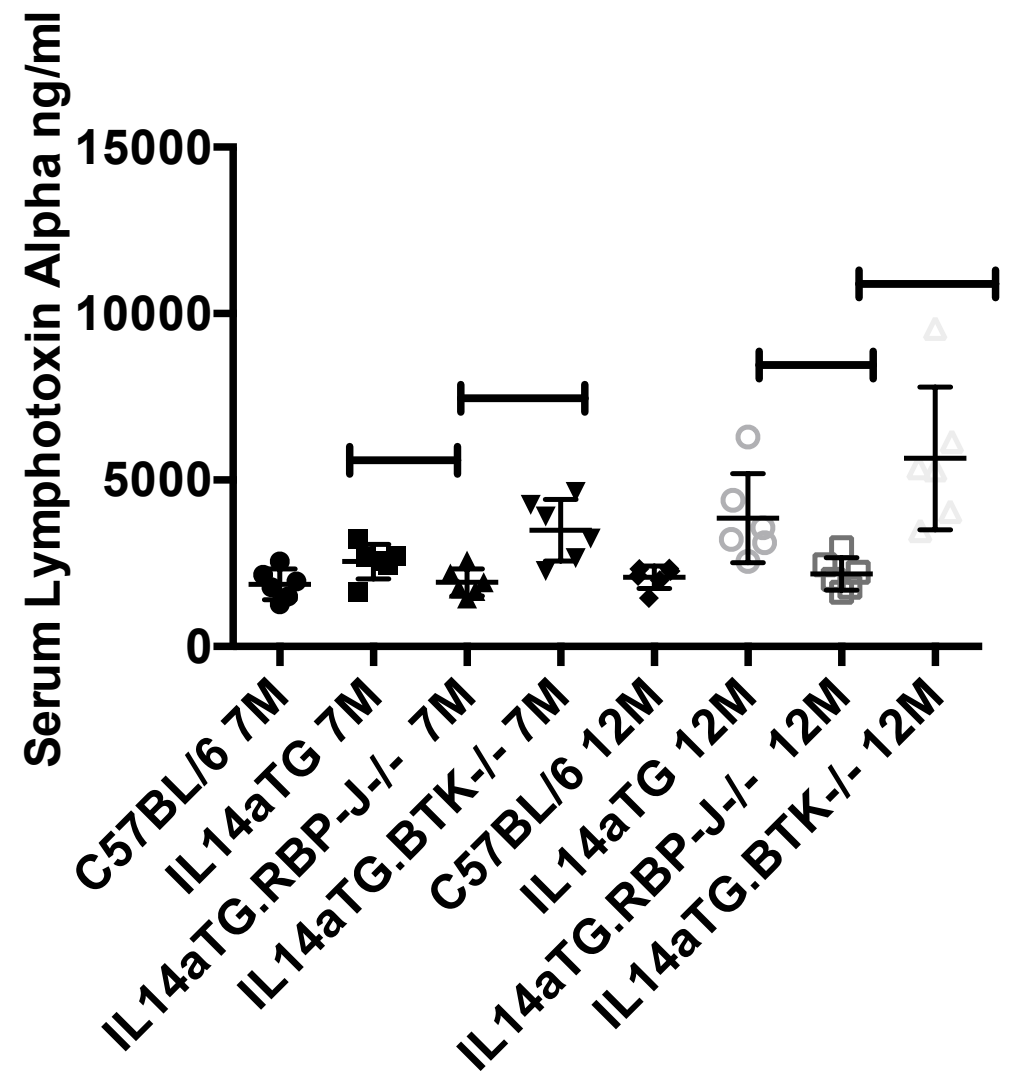


Figure 7

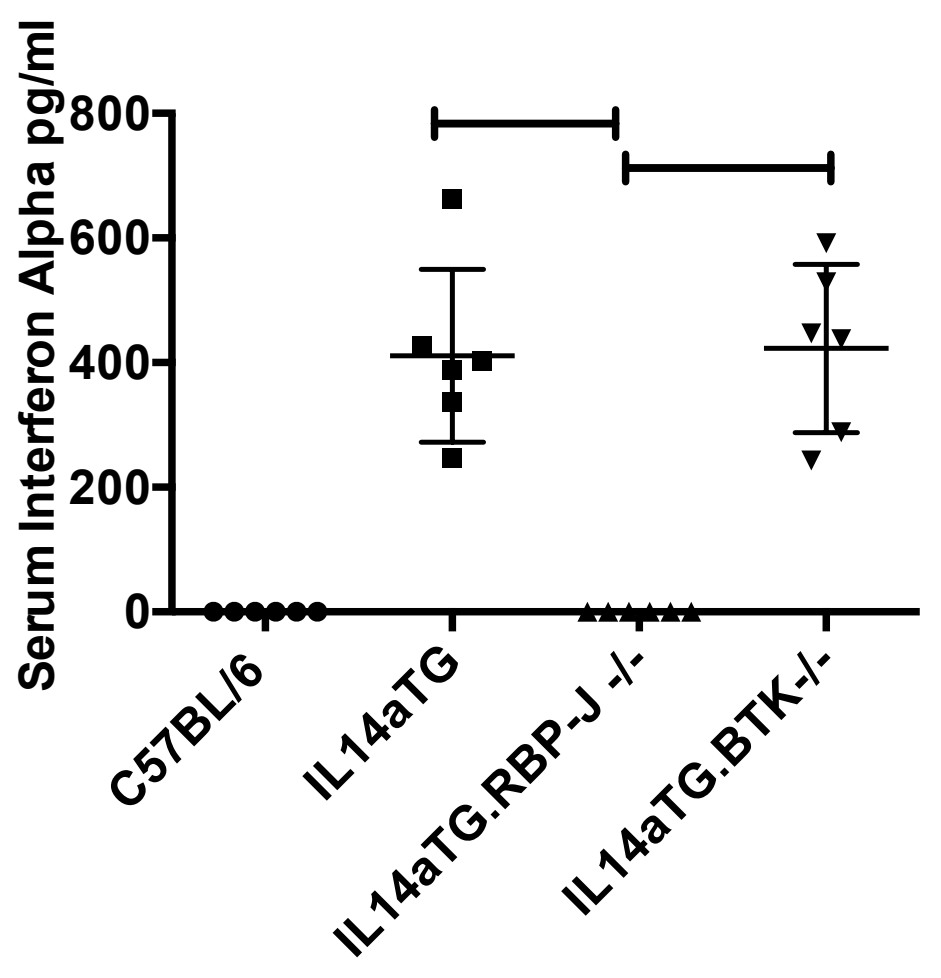

